\title{
Isabelle Durant
}

\section{Entretien avec une eurodéputée verte}

\author{
Recueilli par Delphine Masset
}

\section{Peux-tu nous dire en quelque mot ce que tu fais, qui tu es?}

Député européenne verte, vice-présidente du parlement européen, conseillère communale à Schaerbeek, mère de famille, grand-mère, un peu de tout. Mais plus sérieusement, depuis que je me suis engagée en politique à Écolo (et j’ai toujours été engagée en politique à Écolo), ce qui m'a motivé, c'est la vision systémique de l'ensemble des problématiques. Je ne suis pas entrée par la porte de l'environnement, la porte du multiculturel ou la porte du social mais ce qui m'a toujours motivée c'est vraiment une vision du monde assez globale, une vision dans laquelle l'ensemble des problèmes sont interconnectés et où l'action qu'on mène à un niveau, ici et maintenant, à des répercussions à d'autres. Bref, la question des interactions, l'idée de faire d'une pierre plusieurs coups, d'agir sur plusieurs variables.

Et ce n'est pas un hasard qu'aujourd'hui au Parlement je sois engagée dans les matières que je connais bien c'est-à-dire le transport, le ferroviaire - mais dans une vision service public, dans une vision qui n'est pas une vision technique ou technocratique mais une vision «c'est quoi le transport ferroviaire par rapport à l'ensemble de l'économie, par rapport, aussi à la question des femmes... »

Je suis par ailleurs en budget, qui, par définition, est une matière qui est à la fois un peu technique, mais en même temps une matière qui brasse un ensemble de chose sur les choix qui sont à faire dans chacun des domaines.

Mais aussi sur les questions internationales qui me passionnent et en particulier l'Afrique et le Moyen-Orient qui sont les zones du monde que je connais le mieux. Et où, pour ma part, même si ce n'est peut-être pas de façon saisissante pour tout le monde, il y a évidemment le même type d'analyse et le même type de travail menés sur l'ensemble de ces questions.

Idem d'ailleurs pour ma fonction de vice-présidente pour laquelle je suis attachée à des questions qui touchent à la fois la citoyenneté, la démocratie, le lobbyisme et qui sont des questions qui me préoccupent très très fort... parce qu'elles sont les soubassements de tout le reste en fait!

Donc mon approche écologiste est celle-là. Elle l'a été depuis le début et le reste plus que jamais aujourd'hui, dans mon mandat européen mais aussi à Schaerbeek...

Dans la systémique, il y aussi la question de la cohérence qui me tient à cœur et où je dirais même parfois, je pense que je suis trop honnête parce qu'en politique parfois - je ne dirais pas qu'il faut être malhonnête mais il y a des raccourcis - ou des tournants 
que j'ai du mal à porter. Parce que notre meilleure manière (même si elle n'est pas extrêmement populaire aujourd'hui dans le climat politique et a fortiori sur les questions européennes) d'agir, chez les écologistes, c'est de porter attention à cette cohérence... Je pense que la carte maitresse des écologistes c'est la cohérence.

Et est-ce que tu as l'impression que cette vision systémique t'a amené par exemple à être féministe?

Absolument. Ce sont mes premières batailles. Puisque quand j'avais vingt ans on était dans toute la bataille sur la dépénalisation de l'avortement. Et donc ça a été un de mes premiers combats à la maison des femmes Rue de Méridien à Bruxelles avec une série de femmes qui sont mes aînées mais oui, par définition, la question féministe au sens... à part deux aspects :

(1) Féministe d'une part parce qu'il y a toujours une conquête de droits ou une reconquête de droits ou des batailles contre le détricotage d'une série de droits... On pense à tout ce qui est individualisation des droits, sécurité sociale... Pas seulement la question stricte des tâches ménagères mais tout simplement la question des droits quant à l'aménagement du temps de travail, etc. avec y compris la question de l'avortement parce qu'on sent qu'il y a des pro-vita qui renaissent de leurs cendres. Ce qui se passe en France est très révélateur d'un climat qui revient, que moi je croyais définitivement battu et je dirais que cela appelle à toutes les vigilances et toutes les attentions...

(2) Ça c'est le volet féministe au sens du droit des femmes et puis, il y a le volet que j'appellerais... je n'aime pas le mot « féminin » parce que cela fait penser à travaux de coutures ou de de broderies... mais « féminin » au sens d'une vision plus horizontale des choses. Je crois réellement qu'il y a une manière de faire de la politique - pas une manière dans le sens ou on serait plus gentilles, etc. - mais une approche qui serait plus horizontale, plus réticulaire, dans une logique de réseau, dans une logique de travail où pour aller du point $\mathrm{A}$ au point $\mathrm{B}$, ce n'est pas nécessairement la ligne droite, ce n'est pas nécessairement la confrontation immédiate - ça peut l'être et parfois ça doit l'être. Mais ça peut être aussi des chemins plus détournés, des manières d'aller chercher des alliances à l'extérieur, des manières de travailler sur une logique où on est plutôt avec les gens. Si on doit conclure quelque chose, on peut aussi le conclure dans un chemin qui peut être fait de confrontations mais qui doit aboutir à une forme de compromis et qui aboutit parce qu'on se met à la place de l'autre - un compromis ne se fait pas si il y' a un des deux ou trois acteurs qui perdent leur pantalon. On ne peut pas déshabiller l'autre. L'humiliation en politique il faut l'utiliser à très très très petite dose.

Je pense que dans cette méthode-là (que je ne pense pas forcément féminine) il y’a le volet plus horizontal qui colle très très bien à cette approche systémique qui est la mienne. Moi je me sens comme un poisson dans l'eau là-dedans. Même si ce n'est pas forcément quelque chose qui, aujourd'hui, est plus sexy que l'approche crue, drue sur laquelle on sort et on martèle des slogans ou des actions. Mais je dirais que le siècle qui vient de s'ouvrir est un siècle dans lequel on sera dans une logique plus coopérative, 
dans une logique de travail de réseau, dans quelque chose de beaucoup moins cadré... quelque chose comme " les bons et les méchants ", l'adversaire qu'on pointe et puis quoi ? Je pense que cette manière-là de travailler - je la vis comme étant assez féminine - et je me trouve bien dedans.

Et est-ce que tu as l'impression que les autres députés - plutôt masculins - sont réceptifs à ce type de fonctionnement-là et peuvent reconnaître ces qualités-là ?

Parfois oui, mais il faut pouvoir changer de temps en temps de mode aussi, si on veut se faire entendre ou respecte. Ce n'est pas une question de groupe politique mais c'est aussi une question de profils en politique qui ne sont pas identiques. Il y a des hommes qui travaillent aussi comme moi et vice et versa, il y a des femmes qui sont dans une logique plus guerrière, je n'en fais pas une question sexuée mais plutôt une question de profil. Je pense qui si on veut se faire respecter par un profil guerrier alors qu'on est plus dans un profil réticulaire, il faut aussi de temps en temps pouvoir faire la guerre et démontrer aussi de l'efficacité du système réticulaire par rapport à la stérilité, parfois, de l'approche exclusivement déclarative.

On ne sait pas rester suspendu en l'air. Donc il faut aussi apporter des éléments de réalisation. Bon alors parfois on ne vous l'attribue pas pour d'autres raisons, parce que c'est dans des rapports de forces, dans des familles politiques, etc.

Mais dans la bataille politique globale je pense qu'il faut à la fois amener des résultats, par moment de la confrontation, mais immédiatement la faire suivre du chemin de la solution et ce chemin-là, oui il peut se faire respecter... Ce sont des choses qui sont audibles et entendues, mais je pense que cette méthode-là porte ses résultats.

Et moi dans mon expérience (et ça, ce n'est pas européen) mais je me rappelle pertinemment bien que dans une période de crise, à l'époque de la grève des camionneurs rue de la Loi, et où seule femme (ou presque seule femme au milieu de tous ces hommes), j'avais réussi à faire aboutir le débat... Je pense que c'est un atout d'avoir utilisé cette méthode-là et elle a bien marché. Elle a tellement bien marché que l'accord a été conclu au bout de deux jours et a été exécuté de $\mathrm{A}$ jusqu'à $\mathrm{Z}$. On est dix ans après et les organisations professionnelles du secteur continuent toujours à m'inviter chaque année à leur AG. Donc j'ai gardé une relation étonnante de respect à la fois sur les contenus et les méthodes par des organisations qui étaient loin d'être acquises à l'écologie - les fédérations de transporteurs routiers ce ne sont pas les amis d'Écolo!

Mais parce qu'il y a eu une recherche collective avec tout le monde autour de la table, une recherche d'instruments de solution et sur la loyauté de sa mise en œuvre. On doit être nickel sinon on perd son crédit ! Cette approche-là, elle colle à ma personne mais je pense qu'elle est porteuse de résultats et je pense même qu'à l'échelle européenne, le projet européen est en si mauvais état aujourd'hui - à la fois en mauvais état réel mais aussi en mauvais état dans la perception par bons nombres de citoyens européens, par la superbe dans laquelle parfois il s'est placé, avec une certaine arrogance, etc. - je pense que justement aujourd'hui renouveler (plus que le renouveler, le restaurer sur 
des valeurs qui sont, je dirais, un peu plus égalitaires, etc.) le projet européen passe aussi par là.

Parce qu'il y a deux aspects : il y a les contenus mais il ne suffira pas de revoir les contenus pour convaincre - il faut travailler sur les contenus et sur les méthodes et je pense qu'une méthode plus horizontale avec des échelles de subsidiarité, un plus grand respect pour les entités régionales, pour les parlements nationaux, pour prendre en compte les parlements régionaux qui aujourd'hui n'ont rien à dire... pourrait marcher. Il faudrait décloisonner la question des fonds régionaux de celle de la PAC pour avoir une relation plus réticulaire entre l'UE et les différents niveaux de pouvoirs... et ce n'est pas le centralisme de l'UE contre les États membres bancals et abscons, du siècle dernier... Je pense que cette approche est la seule qui peut remettre l'UE en route. Travailler tout à fait autrement.

C'est intéressant. Je vais quand même, si tu veux bien, revenir sur la question des profils. Estce que tu as l'impression que les femmes sont moins aptes à jouer de manière stratégique, c'està-dire à s'adapter selon les profils réticulaire ou guerrier, selon les situations?

Non au contraire. Parce que les femmes sont plus malines. Enfin, les hommes ne sont pas cons mais d'un côté on joue au billard à cinq bandes et aux échecs - et c'est important car les qualités des joueurs d'échecs sont importantes en politique mais de l'autre, l'approche horizontale est une approche où on doit avoir trois coups d'avance aussi sinon ça veut dire qu'on se défile par rapport à l'objectif qu'on veut atteindre. On sait où on veut aller, le cap est conservé, mais simplement on va chercher, sur des modes différents, des alliances ou des alliés pour reconstruire une position ou regagner un chemin.

Simplement c'est une méthode moins excitante dans un monde où tout est dit de manière courte, rapide et immédiate. Et dans le court, rapide et immédiat, évidemment ce qui est un peu long avec du détour, c'est moins facile à expliquer.

Je vais prendre le débat belge et le débat français. On dit qu'en France c'est bien, au moins c'est gauche-droite, c'est plus clair, on voit bien et en même temps la France est malade, est brouillée, mal avec son régime. On ne peut pas dire que l'affrontement soit la seule manière de percevoir ou de faire percevoir les enjeux d'un problème... Donc moi je plaide pour une appréhension de la complexité vis-à-vis des problèmes. Je suis convaincue que la question de la complexité (qui ne veut pas dire les intellectuels en chambre qui vous élaborent un document incompréhensible) est sur le long terme plus efficace.

Je crois que les écologistes qui sont dans un programme, une vision du monde qui est à la fois complète, globale, systémique mais donc aussi complexe (puisqu'elle intègre l'ensemble des problèmes) doivent pouvoir porter et accepter de porter a fortiori dans ces moments où ce n'est pas facile, la complexité, les contraintes, les adversaires et les alliés, la manière de construire, les alliances - plutôt que d'être exclusivement dans quelque chose qui est porteur et rassurant parce que c'est un message plus court. 
Et je pense que la vision décentralisée qui est la nôtre implique la complexité. Et la complexité ne veut pas dire que les trois quarts des gens ne peuvent pas comprendre, au contraire.

Je voulais revenir sur un élément auquel tu as fait mention, c'est-à-dire les manifestations pro-vie : à quoi associes-tu ce qu'on appelle ce backlash, ce retour du bâton?

Moi je pense que c'est lié à la fois à tout ce qui est mondialisation, libéralisation, libération d'une série de choses et de l'autre côté, je dirais, au retour du religieux... Alors moi je n'ai rien contre le religieux - moi je ne suis pas très religieuse -, mais enfin je n'ai rien contre dans le sens où c'est un bazar qui reste individuel, une relation avec la spiritualité... C'est même extrêmement important que chacun puisse retrouver la question du sens du moins au moins dans son histoire à lui. Mais la montée du religieux instrumentalisée par les partis politiques au sens du retour du prescriptif, du prescriptif social, voire politique... la montée de l'islam ou plutôt d'une certaine forme d'islam... Tout cela ramène ou fait remonter une série de règles ou de limites...

Et donc oui, on vit une période dangereuse à cet égard, je trouve qu'on le pressentait dans les dernières années mais je trouve que là, aujourd'hui, ça nous éclate à la figure et donc on doit être extrêmement attentif à conserver des droits acquis...

Mais on sent bien que la question du religieux, du sens, dans un monde complètement déboussolé et où l'information et l'immédiateté circulent en permanence fait qu'on est dans une société plus puritaine...

La recherche de repère c'est individuel et je dirais la condition d'une vie équilibrée, mais je le vois plus dans une sphère individuelle ou semi-collective, dans des entités choisies, qu'elles soient familiales ou autre y compris dans une relation avec Dieu, pourquoi pas, mais le problème c'est l'instrumentalisation de ça dans la société pour faire une prescription pour tous, identique avec une connotation quasi morale et ça c'est assez dangereux, de mon point de vue...

Est-ce que toi en tant que femme qui fait de la politique tu as pu sentir à un certain moment qu'on te freinait ou qu'il $y$ avait des freins qui étaient dus au fait que tu étais une fermme?

Par vraiment. En plus, moi, j'ai la chance d'avoir hérité d'un capital familial et social extrêmement libre. J'ai été élevée pas du tout comme ma génération par une mère très libérale, très féministe, très ouverte en fait. Pour moi c'était normal et donc il n'y avait pas de raison de prêter le flanc à...

Mais oui j'ai eu pas mal de problèmes, mais des problèmes qui sont liés au parti auquel j'appartiens, à un rapport de force politique, à une certaine inexpérience sur une série de choses à un moment donné mais ça, c'est valable pour un homme. Un homme peut être écrabouillé aussi politiquement dans un rapport de force violent. Mais cette violence je ne l'ai jamais ressentie comme un truc de femme. Et de toute façon dès qu'une blague un peu conne peut sortir, j'ai un registre assez consistant en réponse à... donc ça n’a jamais pris. 
De toute façon, chez les écologistes, même si on ne peut pas dire que les écologistes sont asexués et qu'on ne peut pas dire qu'on est égaux par nature, c'est dans nos principes... C'est quand même beaucoup plus facile chez les écologistes parce qu'on s'est donné des garde-fous, comme sur des questions de durée de mandat, de rétrocession financière... qui obligent à dire " un homme une femme », etc. Parce qu'il ne faut pas croire que les écologistes sont féministes par nature, c'est parce qu'ils se donnent ses règles dans ce domaine-là et, donc, je trouve qu'à Écolo, ce n'est pas très difficile d'exercer un mandat quand on est une femme si on se sent motivée... Mais moi je n'ai jamais vécu ça comme un handicap.

La seule chose que j'ai vu un peu différemment (et à mon avis elle change de la nouvelle génération) c'est que je pense - et de nouveau ça revient à cette approche horizontale - je pense que souvent les femmes en politique quelle que soit la position qu'elle occupe par rapport à leurs pairs masculins... en clair, on ne va jamais demander comment un premier ministre homme fait pour concilier famille et travail... on va toujours aller demander à une femme, quelle bête question! Comment on ne va pas demander à un homme qui travaille $15 \mathrm{~h}$ par jour comment il fait pour concilier sa vie ? Il n'y a personne qui lui demande!

Et c'est vrai que rares sont les ministres qui disent « j'ai oublié de donner le sac de piscine au gamin » et qui va vite téléphoner pour qu'on apporte le sac de piscine. Une femme va faire ça. Je veux dire que, par-là, il y a une sorte d'imputation aux femmes, de, par définition, « c'est plus compliqué pour vous ». Voilà, ça dépend de qui on est, avec qui on vit et comment on s'organise...

C'est d'autant plus injuste que pas mal de femmes sont dans des jobs beaucoup moins gratifiants que la politique - la politique c'est un métier tout à fait passionnant et extrêmement valorisant - valorisant pas seulement dans le sens " on passe à la télévision » mais dans le sens où on contribue quand même à faire avancer les choses (heureusement) à l'inverse beaucoup des femmes sont dans des boulots relativement ingrats, travaillant avec des horaires difficiles, sans compter la précarité... et donc je trouve que c'est assez anormal qu'on vienne nous demander ça à nous qui avons la chance d'avoir un boulot bien payé, valorisant, de qualité (on est un peu crevé mais bon, y'en a d'autres qui sont beaucoup plus crevées avec un boulot pas valorisant, une précarité financière, etc.)

\section{Qu'est-ce qui a fondé ton engagement en politique?}

Difficile à dire... Je pense que c'est un peu dans mon caractère... C'est un peu depuis toujours. J'étais délégué de ma classe quand j'étais à l'école, j’ai toujours été dans cette posture-là. Et par ailleurs mon engagement à Écolo c'est vraiment cette logique d'approche systémique qui m’a motivée et je la combine à des amitiés. J'ai assisté à la naissance d'Écolo. Je veux dire, on habitait en habitat communautaire avec Olivier Deleuze à l'époque... alors voilà, tout ça, c'est un réseau de gens, la politique c'est aussi ça : c'est du réseau d'amitié, du réseau d'engagement... Donc pour moi c'est un peu une seconde 
nature. Parce que je n'ai pas fait de la politique active, j'ai quand même travaillé pendant quinze ans dans tout autre chose... mais donc dans l'enseignement, l'associatif, maison d'éducation prioritaire, discrimination positive... simplement plutôt du point de vue de l'acteur que du décideur - j'étais dans le cadre et puis je me suis mis hors cadre... mais c'est toujours un peu la même chose. Alors voilà, je ne me suis pas dit un jour « je ferai bien de la politique » mais c'est toujours la même logique qui anime les premières batailles... à la fois féministes, multiculturelles à Schaerbeek contre Roger Nol et contre l'extrême droite, etc. Je vois ça de manière tellement fluide que... sauf quand j'ai dû décider de quitter mon boulot pour travailler pour Écolo - là je me suis dit « là je m'engage, je laisse tomber ma nomination dans l'enseignement, je prends un contrat d'emploi donc je prends un risque mais j'ai envie de le prendre » et y compris quand Jacky Morael est venu me chercher je me suis dit « la direction politique d’Écolo, wow».

Il y a encore cette question à laquelle tu as déjà bien commencé à répondre en abordant la systémique : qu'est-ce que les femmes peuvent aujourd'hui apporter à l'écologie?

Je pense que l'écologie est par définition féminine : pas au sens « des femmes » mais au sens de la projection dans l'avenir, à la fois la proximité et la globalité... Bon je pense évidemment qu'hommes et femmes se projettent tous deux dans l'avenir de leur enfant... c'est une évidence... mais je pense que la façon de se projeter ce n'est pas tout à fait la même. Et heureusement d'ailleurs, on n'a quand même pas tout à fait les mêmes fonctions biologiques. Je suis convaincue que le rapport au temps, aux choses qui grandissent est différent entre un homme et une femme... De façon quasi psychologique.

Et c'est pour ça que je pense que l'écologie qui est justement cette dimension du maintenant et du plus tard, de quelque chose où on se projette, où on jette des bases pour la suite... l'écologie c'est très féminin... et ce n'est pas par hasard si quand on regarde le nombre de membres, le nombre de mandataires chez Écolo... ce n'est pas seulement parce qu'on est des bons féministes, parce qu'on a mis en place des règles mais je pense qu'il y a quelque chose qui est un type d'approche différente sans doute de l'approche " gauche classique », c'est à dire qui est une gauche qui est strictement dans une logique de la lutte pour l'égalité point - en omettant et en considérant comme des variables d'ajustements d'autres problèmes... en ayant moins cette vision globale.

Je vais te poser alors une dernière question. C'est intéressant parce que je vois que tu as l'air assez réconciliée par rapport à la question du constructivisme et de l'essentialisme dans le féminisme qui serait de dire "il y a des femmes qui essentiellement, sont différentes par nature et au contraire, finalement, tout ça sont des choses construites socialement et donc il n'y a pas de différences intrinsèques". J'ai l'impression que toi tu es finalement dans l'entre-deux, c'est-à-dire : aujourd'hui, effectivement, en tant que femme, on a pu développer des aptitudes différentes - mais, par ailleurs, elles sont construites socialement... c'est ça? 
Oui, tout à fait et donc c'est sûr qu'il y a, à la fois, des différences objectives et il y a par ailleurs des choses à construire sur lesquelles on peut amener les hommes à entrer dans les modèles qui sont les nôtres et vice et versa. Moi je dirai que tout en me revendiquant féministe dans le sens «le droit des femmes », je vis très bien dans cette espèce d'entre-deux, comme tu dis... je ne me sens ni déchirée, ni écartelée, même si c'est vrai que parfois dans des milieux féministes on dira de moi que je ne le suis pas assez... mais je m'en fou... une fois de plus, ce qui est important c'est d'aboutir ou de faire avancer un sujet ou un autre, une bataille, de lancer une résistance face à un rouleau compresseur qui approche... plus que de savoir si je suis sur une ligne essentialiste ou constructiviste, Dieu sait quoi, moi je navigue... et franchement si ça en dérange d'autres, je dirais « tant pis pour eux, moi ça ne me dérange pas ». 